\title{
Systemic Research of Grid Upscaling on the Numerical Simulation of Oil Reservoir
}

\author{
Ziyi Liu and Jianwei Gu* \\ School of Petroleum Engineering \\ China University of Petroleum \\ Huangdao District, Qingdao city, China, 266580 \\ *Corresponding author
}

\begin{abstract}
Reservoir description needs to transform its geological model to a simulation model by means of dividing grids. Grid upscaling can reduce the number of geologic model's grids, at the same time it can match the real geosphere. For further to see the impact characteristics of grid upscaling on the results of numerical simulation, this paper study it through four respects: the method of grid upscaling, the degree of grid upscaling, the distribution regularity of plane formation parameters and longitudinal formation characteristics. By changing the grid upscaling method, single grid step, the formation plane permeability differential and the geological model of differential between the layers. Finally, we get the systemic research conclusions of grid upscaling on the numerical simulation of oil reservoir.
\end{abstract}

Keywords-grid upscaling; numerical simulation; reservoir parameter; impact analysis

\section{INTRODUCTION}

Reservoir fine description, limited to computer storage and computing cost, has been unable to be widely used in the numerical simulation[1,2]. Since the birth of grid upscaling, due to its maintaining the basic information of original formation, saving the calculation time, improving the efficiency of the numerical simulation work, it has been getting more favors of reservoir engineers[3].

This article studies the upscaling of model's permeability, respectively for the grid upscaling method optimization, grid upscaling degree analysis and the formation of vertical heterogeneity and plane heterogeneity of permeability. Systemic conclusions are given, so we can provide referential materials for other reservoir workers.

\section{RESEARCH OF GRID UPSCALING METHOD}

At present, the widely used upscaling method by reservoir workers can be divided into three categories: Simple average method, composite average method and method based on the flow of three-dimensional numerical solution. This paper selects arithmetic average method (SS), geometric mean method (JH), blend of arithmetic average method (TH-SS) and non-current boundary method (FLD) as representatives for research.

Permeability of the geological model constructed is coarsened respectively by the selected method, results expressed as relative error, upscaling model compared with fine model, as shown in Table 1:
TABLE I UPSCALING MODEL RELATIVE ERROR STATISTICS(\%)

\begin{tabular}{ccccc}
\hline Content & SS & JH & TH-SS & FLD \\
\hline Recovery & 0.81 & 0.76 & 0.76 & 0.76 \\
Water cut & 2.73 & 2.71 & 2.71 & 1.66 \\
$\begin{array}{c}\text { Cumulative } \\
\text { water }\end{array}$ & 0.91 & 0.91 & 0.91 & 0.39 \\
$\begin{array}{c}\text { Production } \\
\text { Average } \\
\text { pressure }\end{array}$ & 0.07 & 0.08 & 0.08 & 0.03 \\
\hline
\end{tabular}

In conclusion, within the scope of the engineering allowable error, you can freely choose upscaling method. And the method based on 3d numerical solution of flow boundary, whose relative error is smaller, is more close to the results of fine description model.

\section{RESEARCH OF THE DEgREE OF GRID UPSCALING}

For the built model, on the basis of fine model, set the grid step length are: $5 \mathrm{~m} \times 5 \mathrm{~m}, 6 \mathrm{~m} \times 6 \mathrm{~m}, 10 \mathrm{~m} \times 10 \mathrm{~m}, 15 \mathrm{~m} \times 15 \mathrm{~m}$, $20 \mathrm{~m} \times 20 \mathrm{~m}, 30 \mathrm{~m} \times 30 \mathrm{~m}$. And compare impacts of different degree of upscaling on the result of numerical simulation. Summarize recovery data of each model, and make it a graph as shown in Figure 1:

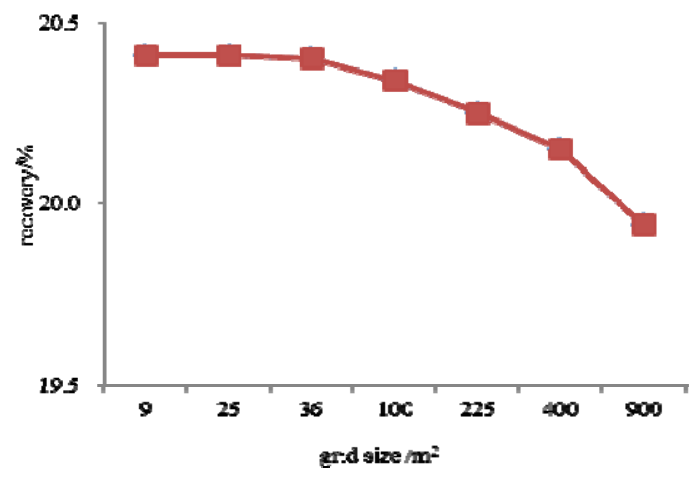

FIGURE I. THE RELATION CURVE BETWEEN THE GRID SIZE AND RECOVERY DEGREE

As shown from the Figure 1, with the increase of grid step, recovery of the model declined.

Water cut curves of different models showed in Figure 2: 


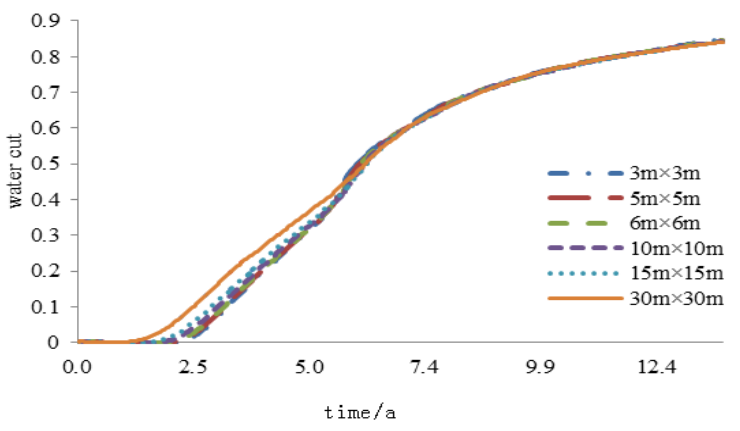

FIGURE II. THE WATER CUT CURVES CHANGE WITH THE DEVELOPMENT TIME

As shown from the Figure 2, when in low and medium water cut period, the bigger the grid step length, at the same time the higher water cut; when in high water cut stage, the grid upscaling degree has less effect on the water cut, whose curves approximately converge.

\section{Research of Plane Heterogeneity on the Results OF NUMERICAL SIMULATION}

By changing the formation plane permeability differential, this paper set different formation conditions including $1,3,6$, 10, 20 five kinds of differential. Recovery datas are shown in Table 2:

TABLE II STATISTICS OF RECOVERY(\%)

\begin{tabular}{cccccc}
\hline $\begin{array}{c}\text { Permeability } \\
\text { differential }\end{array}$ & 1 & 3 & 6 & 10 & 20 \\
\hline $\begin{array}{c}\text { Before } \\
\text { upscaling }\end{array}$ & 16.89 & 16.74 & 16.15 & 15.84 & 15.78 \\
After upscaling & 16.65 & 16.2 & 15.98 & 15.6 & 15.53 \\
\hline
\end{tabular}

As shown from table 2, before and after the upscaling of each model, the greater the permeability differential plane, the lower the recovery.

Select the model of plane permeability differential of 3 and 10 respectively, and compare their water cut - recovery changes, make them into Figure 3 as below:

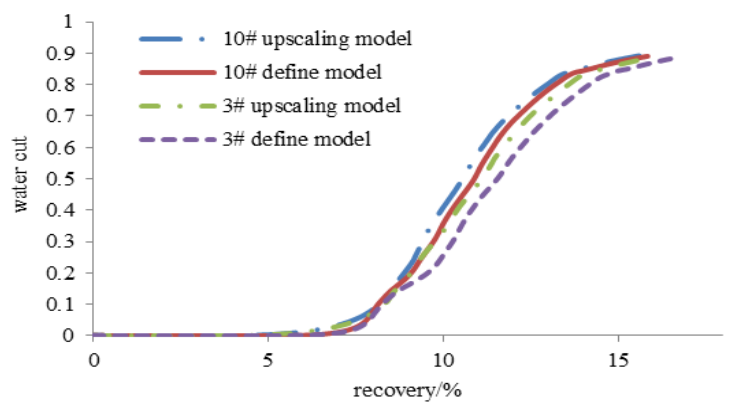

FIGURE III. CURVES OF WATER CUT CHANGES WITH RECOVERY

As seen from the Figure 3, when recovery degree is low, the curve are almost coincidence; when recovery increases, under the same condition, the greater the permeability differential plane, the higher the water cut.

\section{Research of Vertical Heterogeneity on the RESULTS OF NUMERICAL SIMULATION}

Build geological models, under positive and negative rhythm conditions, whose permeability differential between the layers are $1.5,2.3 、 4 、 10 、 20$. Recovery data are shown in Table 3:

TABLE III STATISTICS OF RECOVERY DEGREE(\%)

\begin{tabular}{llllll}
\hline $\begin{array}{l}\text { Permeability } \\
\text { differential }\end{array}$ & 1.5 & 2.3 & 4 & 10 & 20 \\
\hline $\begin{array}{l}\text { Positive-fine } \\
\text { model }\end{array}$ & 20.41 & 20.31 & 20.23 & 19.62 & 19.63 \\
$\begin{array}{l}\text { Negative-fine } \\
\text { model }\end{array}$ & 20.46 & 20.45 & 20.31 & 19.71 & 19.62 \\
$\begin{array}{l}\text { Positive-upscaling } \\
\text { model }\end{array}$ & 20.34 & 20.25 & 20.15 & 19.57 & 19.57 \\
$\begin{array}{l}\text { Negative-upscaling } \\
\text { model }\end{array}$ & 20.46 & 20.45 & 20.37 & 19.77 & 19.67 \\
\hline
\end{tabular}

As shown from the table 3, under the same rhythm and upscaling degrees, the greater the differential between the layers, the lower the recovery; recovery of positive rhythm model is lower than its fine model, and the negative ones are opposite.

\section{CONCLUSIONS}

(1)The method based on 3d numerical solution of flow boundary is more close to the results of fine description model.

(2) The grid step length has a larger influence on low and medium water cut period, but a smaller influence on high water cut stage; grid step length increases, the time of water breakthrough and recovery, in the same conditions, reduces.

(3) The greater the permeability differential plane, the lower the recovery; under the same condition, the greater the permeability differential plane, the higher the water cut.

(4) Under the same rhythm and upscaling degrees, the greater the differential between the layers, the lower the recovery; recovery of positive rhythm model is lower than its fine model, and the negative ones are opposite.

\section{ACKNOWLEDGMENT}

SUSTENTATION FUNDS: National major oil and gas projects " Complex reservoir remaining oil distribution and prediction research investigate " (ZX2011-05009-003).

\section{REFERENCES}

[1] Dai Shuguang. Study on the influence of reservoir numerical simulation research of grid upscaling,June 2012.

[2] Zhu Shaopeng, Zhang Hui, Li Mao. Grid upscaling technology's impact on oil reservoir numerical simulation. China Offshore Oil and Gas, pp.319-321, October 2008.

[3] Li Shuxia, Gu Jianwei. The basis for reservoir numerical simulation[M]. Dongying: China university of Petroleum Press, pp.19-22, 2009. 